\title{
CONSTITUTIVE MODELS AND ARC-LENGTH CONTROL METHOD IN ANALYSIS OF BRANCH-SWITCHING TO BIFURCATION PATH FOR DIAGONAL TENSION FAILURE IN REINFORCED CONCRETE BEAMS
}

\author{
TOSHIAKI HASEGAWA \\ Shimizu Corporation \\ 3-4-17, Etchujima, Koto-ku, Tokyo 135-8530, Japan \\ e-mail: has@shimz.co.jp
}

key words: reinforced concrete, diagonal tension failure, branch-switching, bifurcation analysis

\begin{abstract}
:
The influence of constitutive models for cracking and arc-length control method on analysis of branch-switching to bifurcation path for diagonal tension failure in reinforced concrete beams is examined. Branch-switching from fundamental to bifurcation paths for diagonal tension failure in reinforced concrete beams is performed using eigenvalue analysis and arc-length control method, and the results are compared with those of bifurcation analysis using the direct displacement control method. Distinct first-order eigenmodes corresponding to diagonal and longitudinal cracks are observed in eigenvalue analysis for the branch-switching analysis with the direct displacement control method, but not with the arc-length control method. Due to this fact the analysis with the arc-length control method fails in branch-switching to the bifurcation path for diagonal tension failure. The Multi Equivalent Series Phase Model can evaluate consistent tangential stiffness accurately, and therefore, produces good results in eigenvalue analysis for bifurcation and branchswitching analysis. On the other hand, the secant stiffness has to be utilized in the branch-switching analysis with Multi-directional Fixed Crack Model and Rotating Crack Model due to instability in case of adopting the consistent tangential stiffness, which produces insufficient results in branchswitching analysis.
\end{abstract}

\section{INTRODUCTION}

It is well known that abrupt unstable propagation of diagonal cracks is often observed in the case of diagonal tension failure of reinforced concrete slender beams [1].

To contribute to rational shear safety design of reinforced concrete structures, the mechanism of the failure mode has been investigated through numerical calculations [2], [3], [4]. The author considered the unstable propagation of a diagonal crack as a bifurcation phenomenon from the previous stable cracking state, and tried to simulate the bifurcation path for the diagonal tension failure by using the branch-switching technique with the aid of eigenvalue analysis in a previous study [5]. Although it was shown that the bifurcation analysis was able to simulate reasonably well the mechanism of diagonal tension failure of the slender reinforced concrete beam tested in the experiment, the further diagonal crack propagation into the flexural compression zone in the post-peak regime of structural collapse was not captured well. One of the reasons why 
Table 1: Analysis cases

\begin{tabular}{|c|c|c|c|c|}
\hline $\begin{array}{l}\text { analysis } \\
\text { case }\end{array}$ & $\begin{array}{l}\text { crack constitutive } \\
\text { model }\end{array}$ & path & $\begin{array}{l}\text { bifurcation } \\
\text { point }\end{array}$ & $\begin{array}{l}\text { incremental load } \\
\text { control }\end{array}$ \\
\hline \multirow{4}{*}{ K03 } & \multirow{4}{*}{ MESP } & \multirow{4}{*}{ bifurcation path } & $\mathrm{A}$ & \multirow{4}{*}{$\begin{array}{l}\text { direct displacement } \\
\text { control }\end{array}$} \\
\hline & & & B & \\
\hline & & & $\mathrm{C}$ & \\
\hline & & & $\mathrm{D}$ & \\
\hline L01 & MESP & fundamental path & - & \multirow{9}{*}{$\begin{array}{l}\text { arc-length control } \\
\text { with } \\
\text { indirect displacement }\end{array}$} \\
\hline \multirow{2}{*}{ L02 } & \multirow{2}{*}{ MESP } & \multirow{2}{*}{ bifurcation path } & $\mathrm{A}$ & \\
\hline & & & $\mathrm{B}$ & \\
\hline \multirow{3}{*}{ L03 } & \multirow{3}{*}{ MESP } & \multirow{3}{*}{ bifurcation path } & $\mathrm{A}$ & \\
\hline & & & $\mathrm{B}$ & \\
\hline & & & $\mathrm{C}$ & \\
\hline \multirow{3}{*}{ L04 } & \multirow{3}{*}{ MESP } & \multirow{3}{*}{ bifurcation path } & $\mathrm{A}$ & \\
\hline & & & $\mathrm{D}$ & \\
\hline & & & $\mathrm{E}$ & \\
\hline M01 & MDFC & fundamental path & $\overline{-}$ & \multirow{12}{*}{$\begin{array}{l}\text { direct displacement } \\
\text { control }\end{array}$} \\
\hline M02 & MDFC & bifurcation path & $\mathrm{A}$ & \\
\hline \multirow{2}{*}{ M03 } & \multirow{2}{*}{ MDFC } & \multirow{2}{*}{ bifurcation path } & $\mathrm{A}$ & \\
\hline & & & $\mathrm{B}$ & \\
\hline \multirow{3}{*}{ M04 } & \multirow{3}{*}{ MDFC } & \multirow{3}{*}{ bifurcation path } & $\mathrm{A}$ & \\
\hline & & & $\mathrm{B}$ & \\
\hline & & & $\mathrm{C}$ & \\
\hline \multirow{4}{*}{ M05 } & \multirow{4}{*}{ MDFC } & \multirow{4}{*}{ bifurcation path } & $\mathrm{A}$ & \\
\hline & & & $\mathrm{B}$ & \\
\hline & & & $\mathrm{C}$ & \\
\hline & & & $\mathrm{D}$ & \\
\hline N01 & $\mathrm{RC}$ & fundamental path & - & \\
\hline
\end{tabular}

the post-peak behavior could not be simulated by the bifurcation analysis was considered to be that a direct displacement control method was adopted, which is not suitable for possible snap-back behavior typical in the post-peak regime of shear failure.

In this study on analysis of branchswitching to bifurcation path for diagonal tension failure, the arc-length control method with indirect displacement is adopted as the incremental load control method to overcome the possible post-peak instability of the diagonal crack propagation into the flexural compression zone.

Since the diagonal crack propagation is much influenced by the crack constitutive models adopted in the analysis, the Multidirectional Fixed Crack Model (MDFC Model) and the Rotating Crack Model (RC Model) are utilized in branch-switching analysis, instead of the Multi Equivalent Series Phase Model (MESP Model) used in the previous analysis to examine the influence of crack constitutive models.

\section{ANALYSIS METHOD}

The Multi Equivalent Series Phase Model (MESP Model) as a nonlocal constitutive model and branch-switching analysis functionality have been incorporated into the general purpose finite element system DIANA 9.4 [6], which is utilized in this analysis.

\subsection{Analysis cases}

As shown in Table 1, three series of analysis cases, L, M, and $\mathrm{N}$ are performed in this study, and compared with the previous analysis case $\mathrm{K} 03$, in which the direct displacement control 


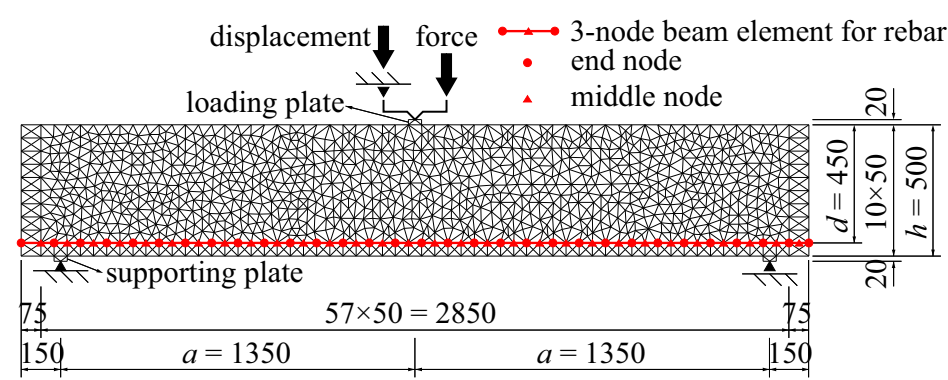

Figure 1: Finite element mesh for concrete and rebar.

was adopted as the incremental load control method, and the MESP Model was used for concrete constitutive model. The arc-length control method with indirect displacement is adopted as the incremental load control method in analysis cases L, and the MESP Model is used as the constitutive model for concrete. On the other hand, in analysis cases $\mathrm{M}$ and $\mathrm{N}$, the MDFC Model and the RC Model are used respectively, along with the direct displacement control method to examine the influence of crack constitutive model on the branch-switching.

\subsection{Analysis models}

As in the previous studies, diagonal tension failure of the slender reinforced concrete beam specimen, BN50, having the effective depth of $450 \mathrm{~mm}$, tested at the University of Toronto [1] is simulated again in this study. The specimen is discretized in linear triangle finite elements of Delaunay triangulation and cross-diagonal meshes for concrete, as well as 3-node beam elements for steel rebar, as shown in Figure 1.

\subsection{Incremental-iterative solution method}

The vertical displacement under the direct displacement control is applied to the central node of steel loading plate elements in analysis cases $\mathrm{M}$ and $\mathrm{N}$ as in K03. On the other hand, analysis cases L adopt indirect displacement control on the same vertical nodal displacement, utilizing the arc-length control method with spherical path constraint.

The regular Newton-Raphson iteration method is utilized to obtain numerical convergence in the numerical iteration process of all analysis cases. The convergence criterion

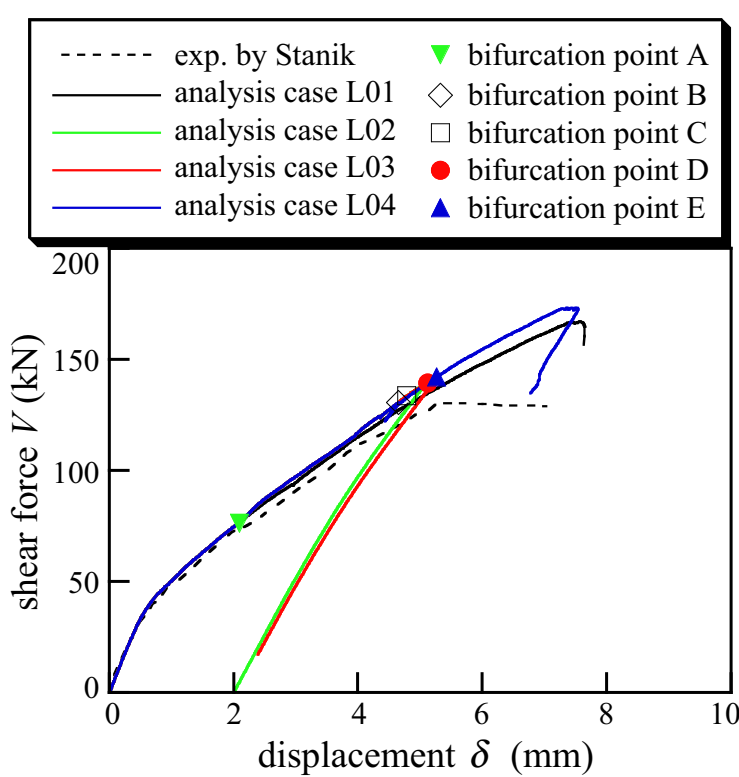

Figure 2: Shear response in analysis case L.

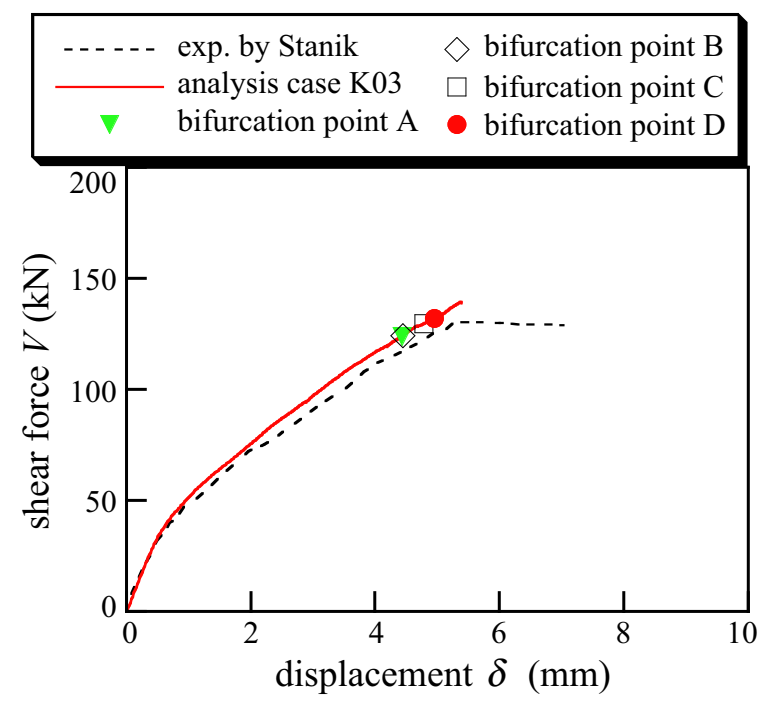

Figure 3: Shear response in analysis case K3.

in case of direct displacement control is based on the Euclidian norm of the out-of-balance force vector. It is assumed that convergence is obtained when the ratio of the norm of outof-balance force vector after each iteration to the norm of the initial value becomes less than the tolerance of $1 \%$. On the other hand, the convergence criterion in case of the arclength control method is based on the energy norm which is composed of internal forces and relative displacements. A convergence tolerance of $0.01 \%$ is adopted in terms of the ratio of energy norm of each iteration to the 


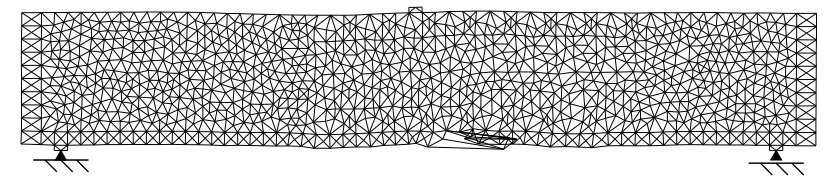

Figure 4: Eigenmode corresponding to spurious kinematic mode in analysis cases L02, L03, and L04.

initial value.

\subsection{Branch-switching method}

To obtain a fundamental path in the shear response of the beam, the equilibrium equation, Eq. 1, is solved by the displacement method of finite element analysis by using direct displacement control or arc-length control with the Newton-Raphson iteration method.

$$
\mathbf{K} \Delta \boldsymbol{u}=\dot{\mu} \boldsymbol{q}
$$

where $\mathbf{K}=$ tangential stiffness matrix; $\Delta \boldsymbol{u}=$ incremental displacement vector; $\dot{\mu}=$ load factor increment; $\boldsymbol{q}=$ normalized load vector. At each load step in the fundamental path a possible bifurcation from the fundamental path to a branch path is checked. If a load step is judged to correspond to a possible bifurcation point, branch-switching from the fundamental path to a bifurcation path is performed. After branch-switching to the bifurcation path, the path is traced by using the same incremental load control method as the fundamental path analysis. When another bifurcation point is detected along the post-bifurcation path, branch-switching is again performed to trace a new bifurcation path. In this study bifurcation point $A$ is first detected, and subsequently bifurcation points $\mathrm{B}, \mathrm{C}, \mathrm{D}$, and $\mathrm{E}$ are found in the following post-bifurcation paths, therefore, branch-switching and tracing post-bifurcation paths are repeated by subsequently following those bifurcation points. Table 1 shows the analysis cases and bifurcation points where branch-switching to bifurcation paths is performed in this study. Figures 2, 3, 24, and 25 show the obtained shear responses and bifurcation points for branch-switching.

In general the bifurcation point and limit point or maximum load are singular points which should be judged by the determinant of the tangential stiffness matrix $\operatorname{det} \mathbf{K}$ of Eq. 2 .

$\operatorname{det} \mathbf{K}=\operatorname{det}\left(\mathbf{L D L} \mathbf{L}^{\mathbf{T}}\right)=\prod_{i=1}^{n} D_{i i}=0$

where $\mathbf{L}=$ lower triangular matrix of $\mathbf{K} ; \mathbf{D}=$ diagonal matrix of $\mathbf{K} ; D_{i i}=$ diagonal element of $\mathbf{D} ; n=$ number of diagonal elements.

Since spurious kinematic modes [7] due to strain softening of concrete material can also produce singular points in the equilibrium equation for concrete structures, emergence of the lowest eigenmode, usually with a negative eigenvalue, corresponding to the spurious kinematic mode is monitored by checking drawn figures of eigenmodes obtained in eigenvalue analysis based on Eq. 3 .

$\mathbf{K} \boldsymbol{v}_{i}=\lambda_{i} \boldsymbol{v}_{i}$

where $\boldsymbol{v}_{i}=$ right eigenvectors of $\mathbf{K} ; \lambda_{i}=$ eigenvalues of $\mathbf{K}$.

The spurious kinematic modes are pathological and mechanically meaningless, and occurrence of the modes results in a premature termination of the numerical analysis. Therefore, bifurcation and limit points are selected from singular points after excluding the spurious kinematic modes, and branch-switching to the bifurcation path is performed starting from the bifurcation or limit points. Figure 4 shows an example for the eigenmode with the eigenvalue $\lambda_{1}=-2.377 \times 10^{1}$, corresponding to a spurious kinematic mode in analysis cases L02, L03, and L04.

An indicator for determinant of tangential stiffness matrix is defined with Eq. 4 to detect possible bifurcation points.

\section{$(-1)^{m} \log _{10}|\operatorname{det} \mathbf{K}|$}

where $m$ is the number of negative pivots in the diagonal matrix $\mathbf{D}$.

The values of $\operatorname{det} \mathbf{K}$ seldom become exactly zero, but turn from positive to negative in the vicinity of bifurcation points as in some buckling problems of structures. In this study the load step where the indicator of $\operatorname{det} \mathbf{K}$ turns from positive to negative is regarded as a singular point or bifurcation point. It might 
be possible to find a point in the path at which the indicator of $\operatorname{det} \mathbf{K}$ gets much closer to zero. However, such precise detection of bifurcation points is not pursued here since incremental load values are chosen small enough compared with the bifurcation load value.

The incremental displacement predictor $\Delta \boldsymbol{u}_{p}$ is calculated from the eigenvector $\boldsymbol{v}_{i}$ with the eigenvalue closest to zero, obtained in eigenvalue analysis and incremental displacement vector $\Delta \boldsymbol{u}_{f}$ as the solution for the fundamental path at the bifurcation point by using Eq. 5. Branch-switching is conducted by applying $\Delta \boldsymbol{u}_{p}$ as a perturbation to obtain a bifurcation path.

$$
\Delta \boldsymbol{u}_{p}=\beta \boldsymbol{v}_{i}=\sqrt{\frac{\Delta \boldsymbol{u}_{f}{ }^{T} \Delta \boldsymbol{u}_{f}}{\boldsymbol{v}_{i}{ }^{T} \boldsymbol{v}_{i}}} \boldsymbol{v}_{i}
$$

$\Delta \boldsymbol{u}_{p}$ of Eq. 5 is derived from the scaling condition, Eq. 6 for $\Delta \boldsymbol{u}_{f}$ and $\Delta \boldsymbol{u}_{p}$.

$$
\Delta \boldsymbol{u}_{f}^{T} \Delta \boldsymbol{u}_{f}=\Delta \boldsymbol{u}_{p}^{T} \Delta \boldsymbol{u}_{p}
$$

\subsection{Constitutive models}

The Multi Equivalent Series Phase Model [8] is used as the concrete constitutive model in analysis cases $\mathrm{K} 03$ and $\mathrm{L}$. The model is based on the Enhanced Microplane Concrete Model [9], and a versatile nonlocal constitutive model, that is capable of describing cracking behavior of concrete under tension as well as shear and compression with good accuracy. The Multi-directional Fixed Crack Model combined with a strain hardening-softening type of elastoplastic constitutive model with the Drucker-Prager criterion under compression is used in analysis cases M. In analysis case $\mathrm{N}$ the total strain formulation type of constitutive model is utilized both for tension and compression, based on the coaxial condition for principal stress and strain axes, which is known as the Rotating Crack Model for tensile cracking.

Although both the MDFC Model and the RC Model are standard crack constitutive models available in DIANA, there are some problems in numerical convergence and instability when consistent crack tangential stiffness is used with regular Newton-Raphson iteration. For this reason it is recommended that the secant stiffness for crack is used as the default in DIANA. In the present research the consistent tangential stiffness has to be used to evaluate appropriate eigenmodes needed in bifurcation analysis, however, the secant stiffness is utilized instead, in order to ensure the numerical stability in cracking problems.

For steel rebar beam elements, an elasticperfectly plastic constitutive model with the Von Mises criterion is assumed, but no plastic yielding occurs in the present analysis.

Perfect bond is assumed between concrete and rebar elements, however, this does not necessarily mean that inelastic debonding behavior of concrete surrounding steel rebar is not taken into account. When rebar elements are in tension a certain shear-tension force is transferred to the surrounding concrete elements, which causes cracking in the elements. The cracking can be regarded as secondary bond cracking which results in inelastic bond behavior.

\section{INCREMENTAL LOAD CONTROL}

\subsection{Influence of arc-length control}

Figure 2 shows the shear responses of analysis cases $\mathrm{L}$ that adopt arc-length control with indirect displacement. Unintentional unloading occurs in analysis cases L02 and L03 although the branch-switching analysis with direct displacement control in analysis case K03 was able to obtain the bifurcation paths that were perturbed by eigenmodes of diagonal cracking. On the other hand, neither the unloading nor the branch-switching to bifurcation path occurs, but a similar shear response to the fundamental path is obtained in analysis case L04. The snap-back behavior after peak load is well captured in analysis case L04 due to the arc-length control.

The first-order eigenmodes corresponding to typical diagonal and longitudinal cracking are not obtained in analysis cases $\mathrm{L}$ utilizing the arc-length control, unlike in the analysis adopting the direct displacement control. Instead, the first-order eigenmodes 


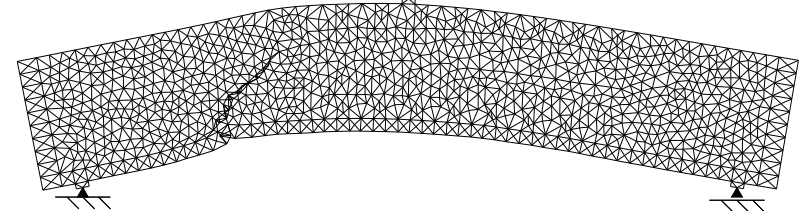

Figure 5: First-order eigenmode as uloading.

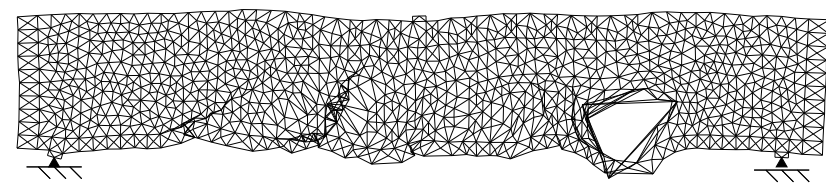

Figure 7: First-order eigenmode at bifurcation point B.

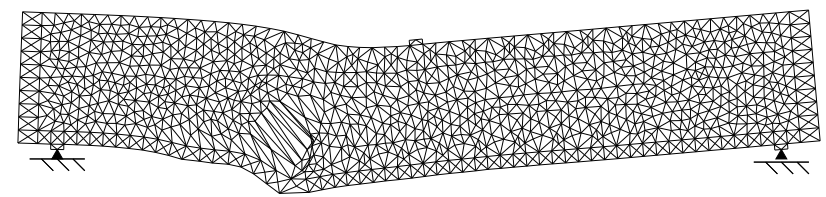

Figure 9: First-order eigenmode at bifurcation point D.

corresponding to unloading behavior with the closure of a diagonal crack are observed more often in the arc-length control as shown in the example in Figure 5. The eigenmode in Figure 5 is drawn with a displacement magnification of 200 , and has the eigenvalue $\lambda_{1}=5.041 \times 10^{1}$. The branch-switching is not done when the first-order eigenmode corresponding to unloading is obtained at the bifurcation point since the diagonal crack propagation is suppressed and unloading occurs actually if the perturbation of the eigenmode was applied.

Figures 6-10 are the eigenmodes for eigenvectors $\boldsymbol{v}_{i}$ chosen to calculate the incremental displacement predictor $\Delta \boldsymbol{u}_{p}$ for perturbation, in which the displacement is enlarged with a magnification of 200 . The individual eigenvalues for bifurcation points $\mathrm{A}, \mathrm{B}, \mathrm{C}, \mathrm{D}$, and $\mathrm{E}$ are $\lambda_{1}=1.632 \times 10^{1}$, $\lambda_{1}=1.242 \times 10^{1}, \quad \lambda_{5}=7.089 \times 10^{3}, \quad \lambda_{1}=$ $-3.892 \times 10^{-2}$, and $\lambda_{1}=1.407 \times 10^{1}$, respectively.

Except for bifurcation point $\mathrm{C}$ the first-order or lowest eigenmodes are utilized. Figures $11,12,13,14$ and 15 show the incremental displacement at load steps just after bifurcation points $\mathrm{A}, \mathrm{B}, \mathrm{C}, \mathrm{D}$, and $\mathrm{E}$ in the post-bifurcation paths, which are immediately after branch-

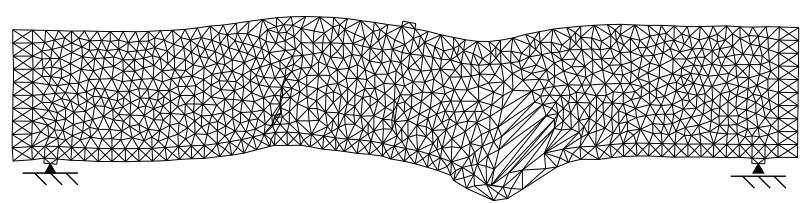

Figure 6: First-order eigenmode at bifurcation point A.

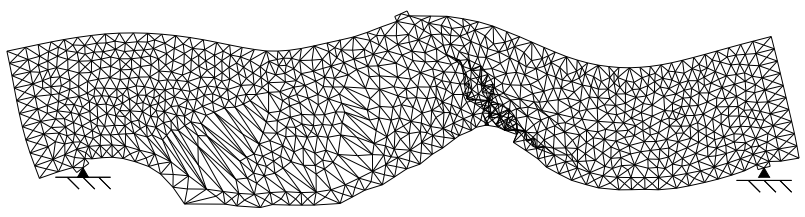

Figure 8: Fifth-order eigenmode at bifurcation point $\mathrm{C}$.

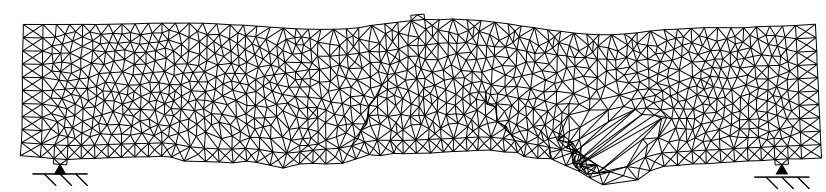

Figure 10: First-order eigenmode at bifurcation point E.

switching. The distribution of maximum principal strain $\varepsilon_{1}$ is superimposed in those figures. Widening and further propagation of the main diagonal and longitudinal cracks along steel rebar are not induced by the branch-switching at all bifurcation points. On the contrary, the unloading takes place after bifurcation point $\mathrm{E}$.

Figures 16, 17, 18, and 19 show the incremental displacement with maximum principal strain at the steps for the maximum shear load in analysis cases L01, L02, L03, and L04. The divergence occurs just after the maximum shear load in analysis case L01 for the fundamental path, but the snap-back response is captured very well in analysis case L04. On the other hand, in analysis cases L02 and L03, the branch-switching results in the paths for unloading finally, and the limit load for diagonal tension failure is not attained.

It is considered that there are lots of possible equilibrium paths for various fracture modes in the vicinity of a bifurcation point in case of fracture analysis of reinforced concrete structures. Branch-switching analysis utilizing the arc-length control method, which is combined with selected constraint conditions such as the spherical path constraint herein, 


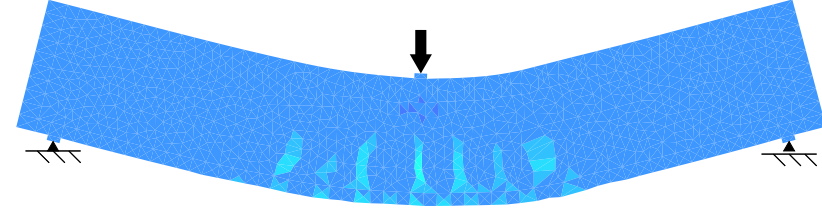

Figure 11: Incremental displacement after bifurcation point A in analysis case L02, L03, and L04.

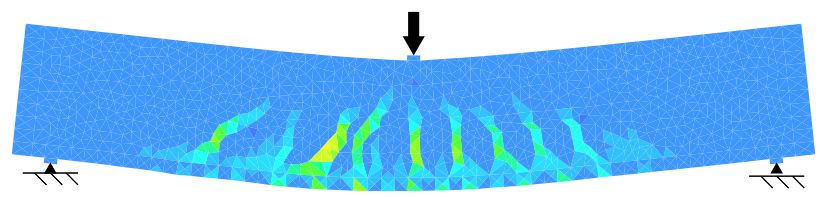

Figure 13: Incremental displacement after bifurcation point $\mathrm{C}$ in analysis case $\mathrm{L} 03$.

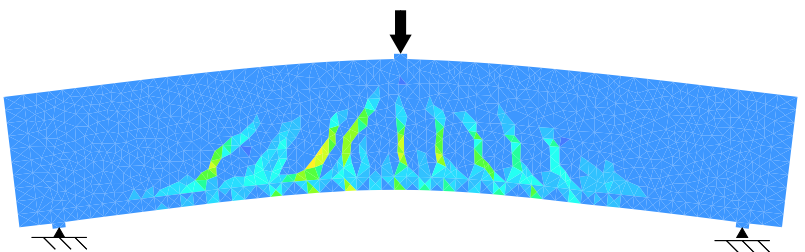

Figure 15: Incremental displacement after bifurcation point $\mathrm{E}$ in analysis case L04.

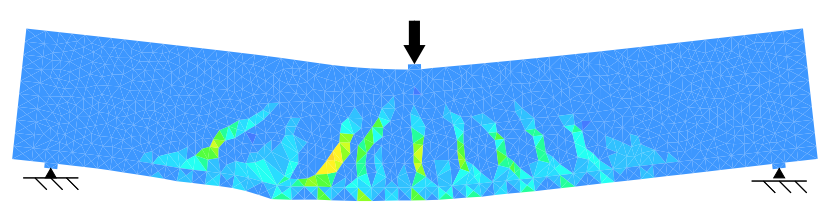

Figure 17: Incremental displacement at maximum shear load in analysis case L02.

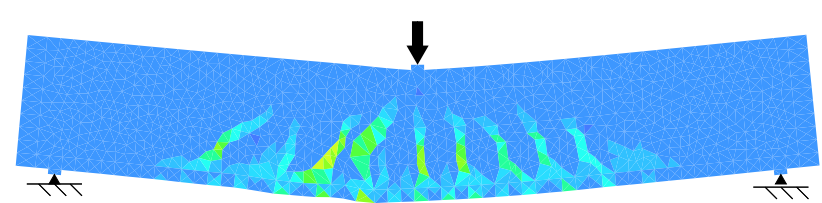

Figure 12: Incremental displacement after bifurcation point B in analysis case L02 and L03.

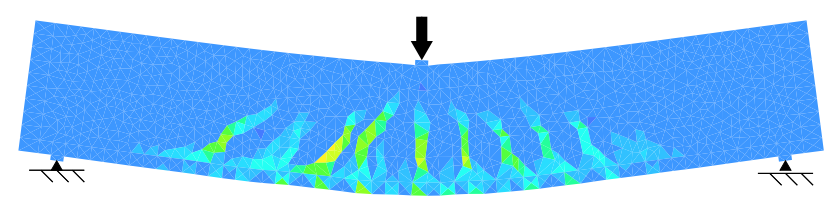

Figure 14: Incremental displacement after bifurcation point D in analysis case L04.

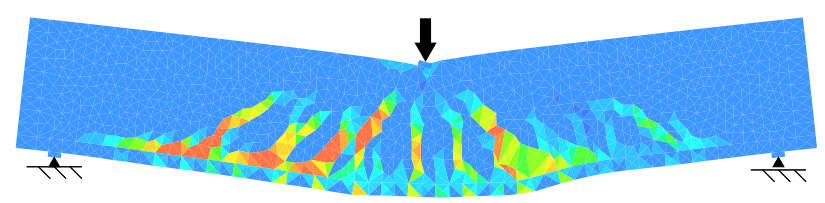

Figure 16: Incremental displacement at maximum shear load in analysis case L01.

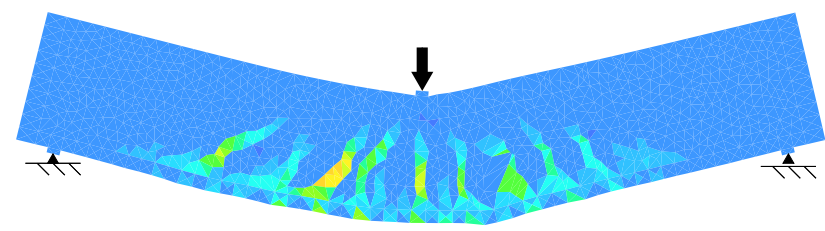

Figure 18: Incremental displacement at maximum shear load in analysis case L03.

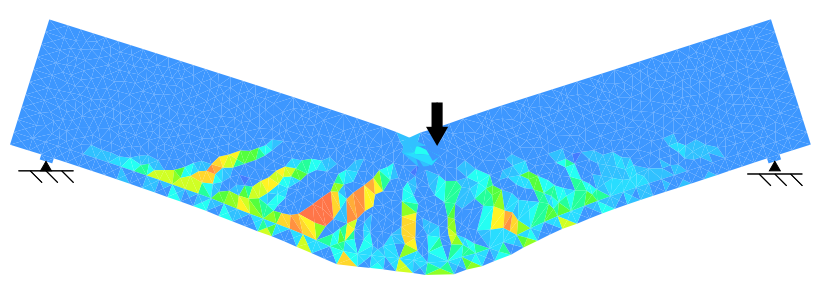

Figure 19: Incremental displacement at maximum shear load in analysis case L04.

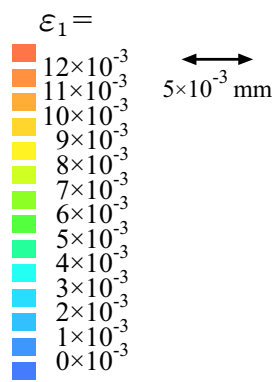

the adaptive loading method, the loadingunloading criterion, the indirect displacement control method, automatically searches and selects a single path among the various possible equilibrium paths for fracture modes in the vicinity of the bifurcation point. Such an automatically selected bifurcation path is not always necessarily consistent with the first- order eigenmode applied as a perturbation, and then the appropriate diagonal tension failure mode is not obtained. Sudden unloading responses occur easily with the arc-length control method, as pointed out in the past research [2]. The results in the unloading responses seem to be due to the fact that the first-order eigenmodes corresponding to 


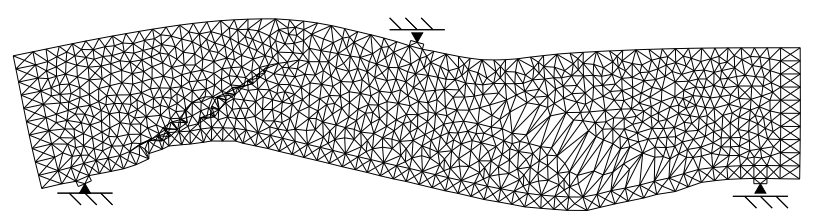

Figure 20: First-order eigenmode at bifurcation point D.

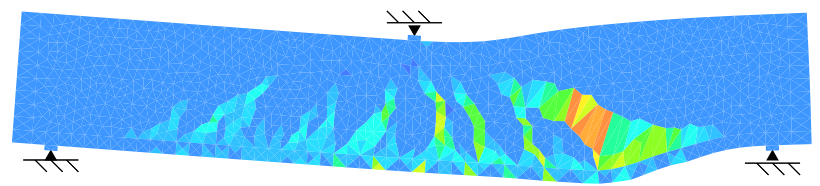

Figure 22: Incremental displacement at maximum shear load in analysis case K03.

unloading emerges frequently in the eigenvalue analysis.

Although the arc-length control method with indirect displacement might be able to capture the unstable fracture in the post-peak snap-back behavior, there are some difficulties in applying the method to the analysis of branch-switching to the bifurcation path for diagonal tension failure by using perturbation of eigenmodes corresponding to the localized fracture.

\subsection{Influence of direct displacement control}

Figure 20 is the eigenmode for eigenvector $\boldsymbol{v}_{i}$ chosen to calculate the incremental displacement predictor $\Delta \boldsymbol{u}_{p}$ for perturbation in analysis case K03 utilizing the direct displacement control method, in which the displacement is enlarged with a magnification of 200. The eigenvalue for bifurcation point $\mathrm{D}$ is $\lambda_{1}=3.6131 \times 10^{2}$ of the first-order, or lowest eigenmode.

Figure 21 shows the incremental displacement at the load step just after bifurcation point $\mathrm{D}$ in the post-bifurcation path, which is immediately after branch-switching. The distribution of maximum principal strain $\varepsilon_{1}$ is superimposed in the figure. Widening and further propagation of the main diagonal and longitudinal cracks along steel rebar in the right span of the beam are induced by the branch-switching at bifurcation point $\mathrm{D}$, which utilizes the lowest eigenmode corresponding to the propagation mode of the main diagonal

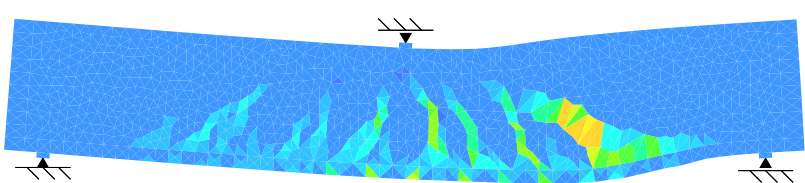

Figure 21: Incremental displacement after bifurcation point $\mathrm{D}$ in analysis case $\mathrm{K} 03$.

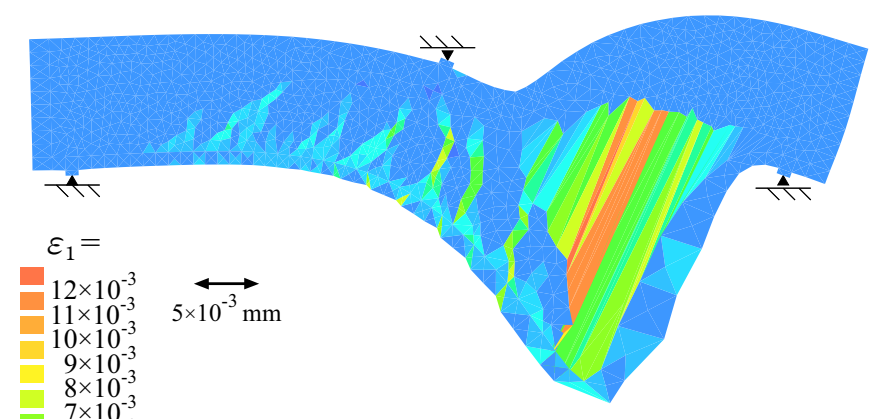

Figure 23: Incremental displacement in post peak in analysis case K04.

and longitudinal cracks. Figures 22 and 23 for analysis case K03 show the incremental displacement with maximum principal strain at the steps for the maximum shear load, and in the post-peak regime. In the case of utilizing the direct displacement control method, the branch-switching to bifurcation paths at bifurcation points $\mathrm{B}, \mathrm{C}$, and D obviously produces drastic propagation of the main diagonal and longitudinal cracks prior to the limit load, which indicates the complete mechanism of diagonal tension failure as well as the collapse process of the shear beam.

\section{CONSTITUTIVE MODEL}

Figures 24 and 25 show the obtained shear responses and bifurcation points for branch-switching in analysis cases $M$ for the MDFC Model, and N for the RC Model. The maximum shear strength of the reinforced concrete beam is slightly underestimated with the MDFC Model, but substantially underestimated with the RC Model. The shear stiffness of the beam is much smaller for the RC Model than the experiment, but is evaluated with good accuracy with the MDFC Model.

The first bifurcation point $\mathrm{A}$ is detected in 

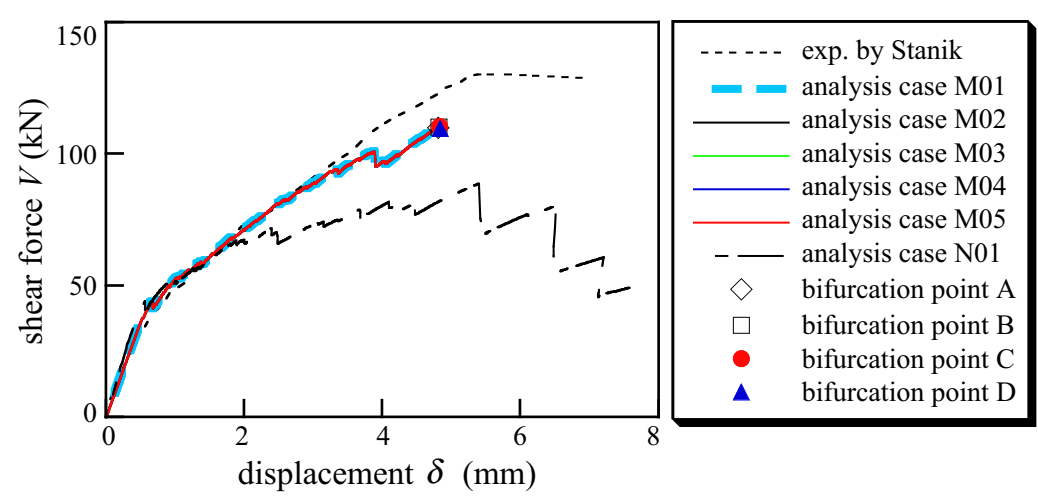

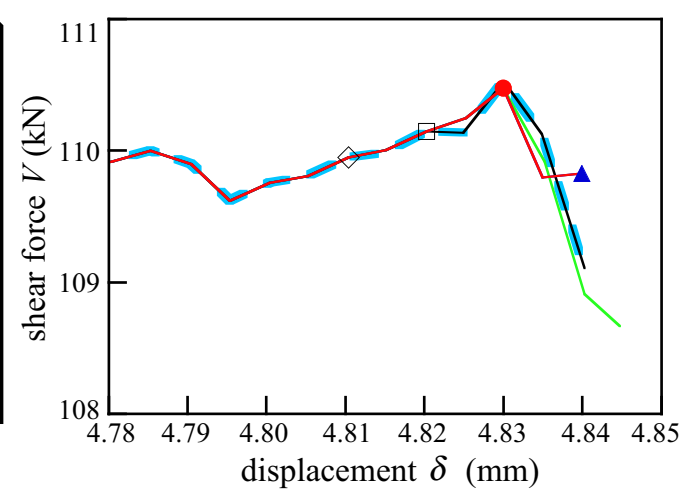

Figure 25: Bifurcation points in analysis case $\mathrm{M}$.

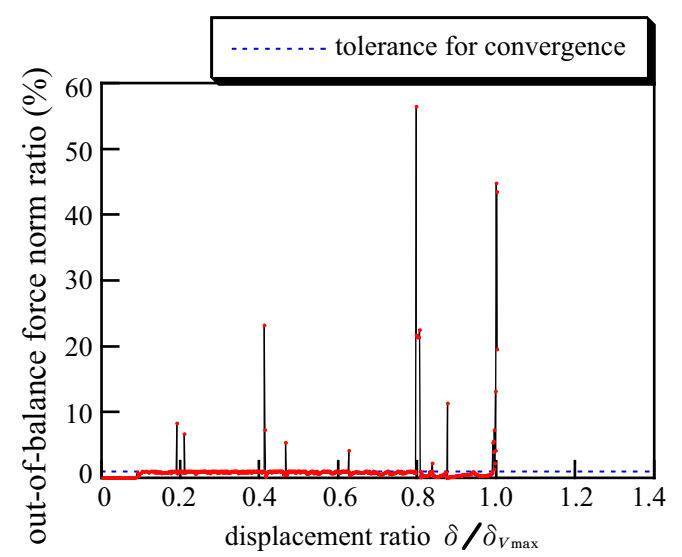

Figure 27: Out-of-balance force norm ratio in analysis case M01.

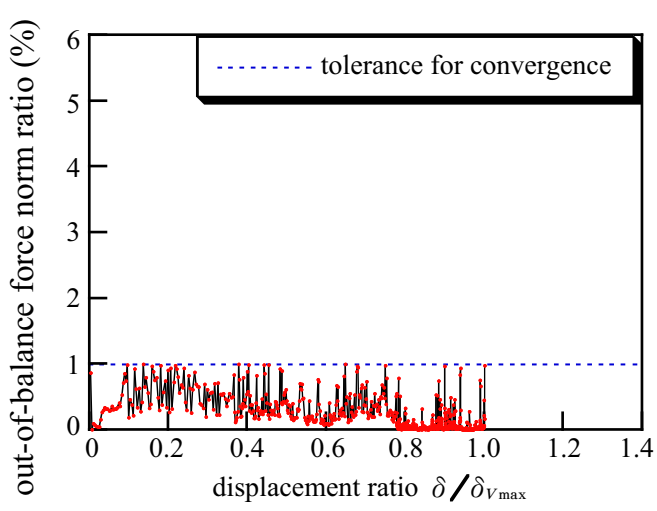

Figure 29: Out-of-balance force norm ratio in analysis case K03.

number of bifurcation points is very small in analysis cases $\mathrm{M}$ and there are no bifurcation points in analysis case $\mathrm{N}$ is because the secant stiffness in the crack constitutive relations is utilized to perform eigenvalue analysis.

Figures 27, 28, and 29 show the out-ofbalance force norm ratio at the end of iteration in analysis cases M01 with the MDFC Model, 


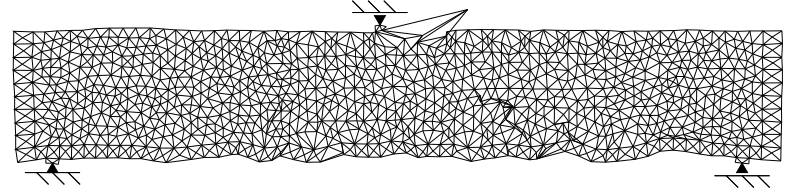

Figure 30: Eigenmode corresponding to spurious kinematic mode in analysis cases $M$.

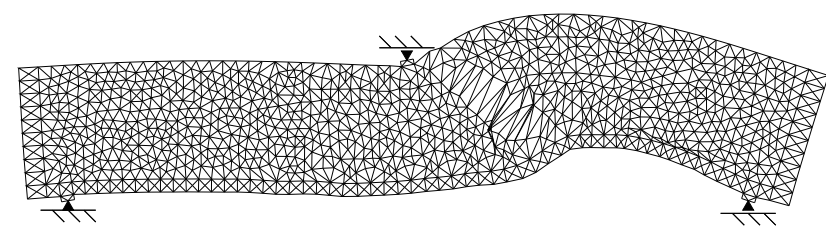

Figure 32: Second-order eigenmode at bifurcation point $\mathrm{A}$.

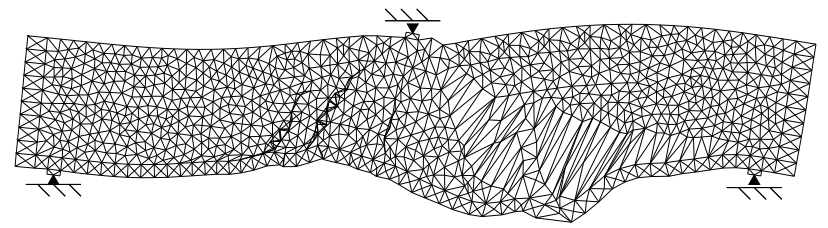

Figure 34: Fourth-order eigenmode at bifurcation point A.

N01 with the RC Model, and K03 with the MESP Model. The out-of-balance force norm ratio cannot decrease to less than the tolerance for convergence of $1 \%$ after the maximum number of iterations at many steps in analysis cases M01 with the MDFC Model, N01 with the RC Model, however, the calculations are continued to the next step, bringing the out-ofbalance forces in the final iteration to the next step, although strictly speaking the calculation should be terminated. The convergency of iterative calculation for the MDFC Model and the RC Model is not that good, despite using the secant stiffness for crack constitutive relations to avoid the instability with softening stiffness. On the other hand, the convergence criterion is satisfied at all the steps in analysis case K03 with the MESP Model, which confirms that MESP Model has excellent convergency and robustness as a numerical crack model.

Figure 30 is the only one eigenmode corresponding to a spurious kinematic mode that emerges in analysis cases $M$ with the MDFC Model. The spurious kinematic mode appears just before bifurcation point $\mathrm{A}$, and

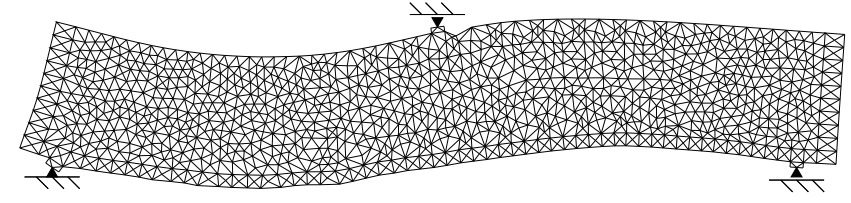

Figure 31: First-order eigenmode at bifurcation point A.

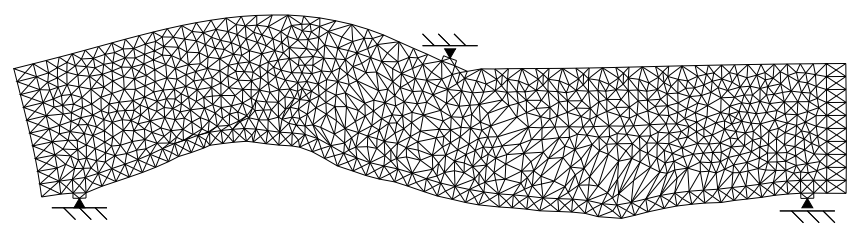

Figure 33: Third-order eigenmode at bifurcation point A.

its eigenvalue $\lambda_{1}$ is $-1.3089 \times 10^{2}$. Spurious kinematic modes do not easily emerge because the secant crack stiffness is used in analysis cases $\mathrm{M}$. The branch-switching to bifurcation path is performed starting from bifurcation point A by applying $\Delta \boldsymbol{u}_{p}$ of Eq. 5 as a perturbation to obtain the bifurcation path. As shown in Figure 25, the branch-switching is performed at bifurcation points $\mathrm{A}, \mathrm{B}$, and $\mathrm{C}$, however, the shear responses on the postbifurcation paths do not differ much from the response on the fundamental path.

Figures 31, 32, 33, and 34 are the first-, second-, third-, and fourth-order eigenmodes at bifurcation point $\mathrm{A}$, and the eigenvalues of the eigenmodes are $\lambda_{1}=5.2820 \times 10^{2}, \lambda_{2}=$ $1.4975 \times 10^{3}, \quad \lambda_{3}=2.0419 \times 10^{3}$, and $\lambda_{4}=$ $5.0663 \times 10^{3}$, respectively. The second bifurcation point $\mathrm{B}$ is on the post-bifurcation path after the branch-switching at bifurcation point $\mathrm{A}$ by applying the perturbation adopting the first-order eigenmode of bifurcation point $\mathrm{A}$. The first-order eigenmodes at bifurcation point $B$ as well as $C$ and $D$ resemble the one at the first bifurcation point $\mathrm{A}$. This is consistent with the fact that the post-bifurcation paths from bifurcation points $\mathrm{B}, \mathrm{C}$, and $\mathrm{D}$ do not much differ from the fundamental path.

Figures 35 and 36 show the incremental displacement at load steps just after bifurcation points $A$ and $B$ in the post-bifurcation paths, which are immediately after applying perturbations in analysis cases $M$. The distibution of maximum principal strain $\varepsilon_{1}$ 


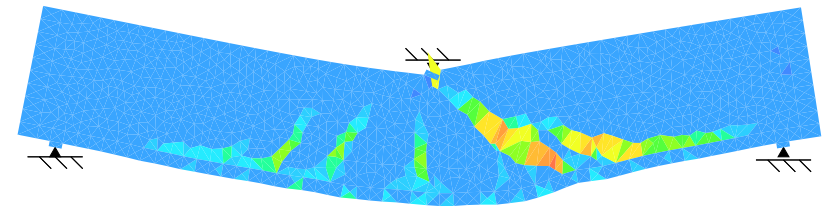

Figure 35: Incremental displacement after bifurcation point $\mathrm{A}$ in analysis cases M02, M03, M04, and M05.

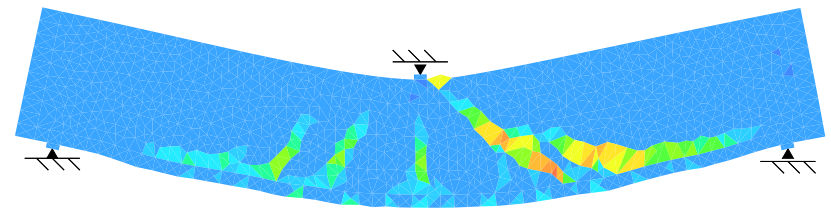

Figure 36: Incremental displacement after bifurcation point B in analysis cases M03, M04, and M05.

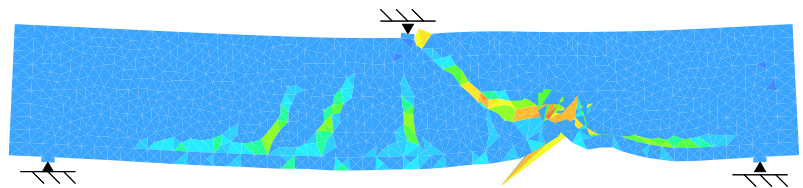

Figure 37: Incremental displacement at maximum shear load in analysis case M05.

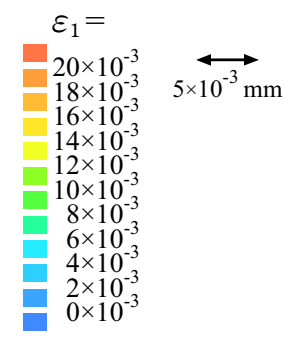

is superimposed in those figures. The overall flexural deformation of beam is much dominant in Figures 35 and 36 despite the branchswitching, although the influence of the first-, second-, third-, and fourth-order eigenmodes appear in the incremental displacement, which is recognized as the failure of compressive fiber concrete in the vicinity of the loading plate, the diagonal crack, and longitudinal crack.

Figures 37 and 38 for analysis cases M05 and N01, respectively utilizing the MDFC Model and the RC Model, show the incremental displacement along with the maximum principal strain at the steps for the maximum shear load. The dominant crack in analysis case M05 with the MDFC Model has a straight shape rather than the curved shape of the diagonal cracks observed in experiments. The failure mode seems to represent a shear compressive failure or a flexural shear failure mode, although shear sliding deformation at the dominant diagonal crack is distinguished and the compressive failure occurs in the vicinity of the loading plate. On the other hand, the shear cracks in analysis case N01 with the RC Model have bent shapes at the tip area, due to the constitutive formulation based on the coaxial condition for principal stress and strain axes in the crack model, and the analysis does not simulate the mechanism of diagonal tension failure.

\section{CONCLUSIONS}

Analysis of branch-switching to bifurcation path for diagonal tension failure in a reinforced concrete beam is performed to study the influence of crack constitutive models and the incremental load control method on the bifurcation analysis.

Since the eigenmode for unloading is more dominant rather than diagonal and longitudinal cracking in the analysis utilizing the arc-length control method with indirect displacement, bifurcation path for diagonal tension failure is not obtained in the analysis.

Eigenmodes corresponding to diagonal tension failure are distinguished in the analysis utilizing the direct displacement control method, therefore, the branch-switching to bifurcation path for the failure is achieved.

The Multi Equivalent Series Phase Model can produce good results in eigenvalue analysis for bifurcation and branch-switching analysis, since the model can evaluate consistent tangential stiffness accurately, and has good convergency.

Secant stiffness has to be utilized in the branch-switching analysis with Multidirectional Fixed Crack Model and Rotating Crack Model due to instability in case of adopting the consistent tangential stiffness, which produces insufficient results in branchswitching analysis. 


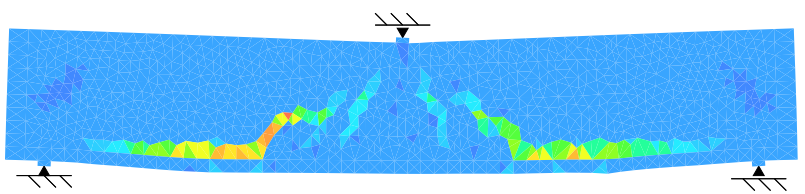

Figure 38: Incremental displacement at maximum shear load in analysis case N01.

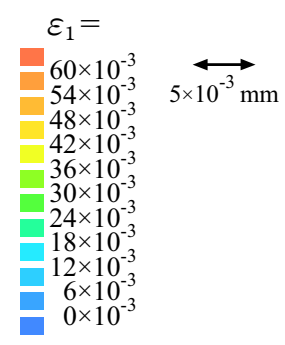

\section{REFERENCES}

[1] Podgorniak-Stanik, B. A., 1998. The influence of concrete strength, distribution of longitudinal reinforcement, amount of transverse reinforcement and member size on shear strength of reinforced concrete members. M.A.S. thesis, University of Toronto.

[2] Hasegawa, T., 2004. Numerical study of mechanism of diagonal tension failure in reinforced concrete beams. In V. C. Li, C. K. Y. Leung, K. J. Willam, and S. L. Billington (eds), Fracture Mechanics of Concrete Structures, Proceedings of the Fifth International Conference on Fracture Mechanics of Concrete and Concrete Structures, Vol. 1, Ia-FraMCoS, USA; pp.391-398.

[3] Hasegawa, T., 2007. Finite element analysis of diagonal tension failure in $\mathrm{RC}$ beams. In A.Carpinteri, P.G. Gambarova, G. Ferro, and G. A. Plizzari (eds), Fracture Mechanics of Concrete and Concrete Structures - Design, Assessment and Retrofitting of RC Structures, Proceedings of the Sixth International Conference on Fracture Mechanics of Concrete and Concrete Structures, Vol. 2, Taylor \& Francis Group, UK; pp.709-717.

[4] Hasegawa, T., 2010. Rebar bond slip in diagonal tension failure of reinforced concrete beams. In B. H. Oh, O. C. Choi, and L. Chung (eds), Fracture Mechanics of Concrete and Concrete Structures - Assessment, Durability, Monitoring and Retrofitting of Concrete Structures, Proceedings of the Seventh International Conference on Fracture Mechanics of Concrete and Concrete Structures, Vol. 2, Ia-FraMCoS, Korea; pp.786-793.
[5] Hasegawa, T., 2013. Branch-switching to bifurcation path for diagonal tension failure of reinforced concrete beam, based on eigenvalue analysis. In J.G.M. Van Mier, G. Ruiz, C. Andrade, R.C. Yu, and X.X. Zhang (Eds), Fracture Mechanics of Concrete and Concrete Structures, Proceedings of the Eighth International Conference on Fracture Mechanics of Concrete and Concrete Structures, IaFraMCoS, Spain; 352.

[6] Manie, J., and Kikstra, W. P., 2010. DIANA - Finite Element Analysis, User's Manual release 9.4.3, TNO DIANA BV, The Netherlands.

[7] De Borst, R., 1989. Analysis of spurious kinematic modes in finite element analysis of strain-softening solids. In J. Mazars and Z. P. Bazant (eds), Cracking and Damage: Strain Localization and Size Effect, ELSEVIER Science Publishers, UK; pp.335-345.

[8] Hasegawa, T., 1998. Multi equivalent series phase model for nonlocal constitutive relations of concrete. In $\mathrm{H}$. Mihashi and K. Rokugo (eds), Fracture Mechanics of Concrete Structures, Proceedings of the Third International Conference on Fracture Mechanics of Concrete Structures, Vol. 2, AEDIFICATIO Publishers, Freiburg; pp.1043-1054.

[9] Hasegawa, T., 1995. Enhanced microplane concrete model. In F. H. Wittmann (ed), Fracture Mechanics of Concrete Structures, Proceedings of the Second International Conference on Fracture Mechanics of Concrete Structures, Vol. 2, AEDIFICATIO Publishers, Freiburg; pp.857-870. 